\title{
Tumor-derived exosomes induced M2 macrophage polarization and promoted the metastasis of osteosarcoma cells through Tim-3
}

\section{Zhonghua Cheng}

Huanggang Central Hospital

\section{Liqin Wang}

Huanggang Centrak Hospital

Chenhuan Wu

Huanggang Central Hospital

Lin Huang

Huanggang Cental Hospital

\section{Yuan Ruan}

Huanggang Central Hospital

Wei Xue ( $\triangle$ xueweixw361@163.com)

Huanggang Central Hospital

\section{Research}

Keywords: Exosomes, osteosarcoma, Tim-3, metastasis, TAM

Posted Date: February 13th, 2020

DOl: https://doi.org/10.21203/rs.2.23484/v1

License: (1) This work is licensed under a Creative Commons Attribution 4.0 International License. Read Full License

Version of Record: A version of this preprint was published on November 5th, 2020. See the published version at https://doi.org/10.1016/j.arcmed.2020.10.018. 


\section{Abstract}

Background: Osteosarcomas, the second most prevalent primary malignancy of the bone, are often presented with high-grade subclinical metastatic disease that metastasizes at very early stages. Exosomes, as molecular information carriers, may play a potent role in the occurrence and development of tumors through oncogenic molecular reprogramming of tumor-associated macrophage (TAM). In this study, we will investigate the effect of osteosarcoma-derived exosomes on the polarization of TAM and decipher its underlying molecular mechanism. Material and

Methods: Osteosarcoma-derived exosomes from MG63 cells were isolated and characterized by transmission electron microscopy, and nano-particle size analysis. Double fluorescence staining was performed to confirm the macrophages phagocytosis of exosomes. Western blot, qRT-PCR, and transwell assays were conducted to assess the effect of exosomes on migration, invasion, and macrophage differentiation. The mouse model of osteosarcoma was established to evaluate the effects of exosomes on lung metastasis in vivo.

Results: MG63 exosomes were successfully isolated and verified to be phagocytized by macrophages through fluorescence confocal microscopy. The results revealed that osteosarcoma cells could induce M2 type differentiation of macrophages largely through Tim-3 mediated by exosomes, which in turn could promote the migration, invasion, EMT, and lung metastasis of osteosarcoma cells through the secretion of cytokines including IL-10, TGF $\beta$, and VEGF.

Conclusions: Our results demonstrated that osteosarcoma-derived exosomes induced M2 polarization of macrophages and promoted the invasion and metastasis of tumors through Tim-3; besides, the study also suggests a novel therapeutic target for future studies.

\section{Background}

Osteosarcoma represents the fourth most frequent malignant primary neoplasm of the bone, constituting approximately $35 \%$ of all the primary malignant bone tumors. Predominantly, osteosarcomas originate in the metaphyseal region of tubular long bone during adolescence and early adulthood and are histologically characterized by the presence of malignant mesenchymal cells producing osteoid or immature bone. Approximately $15 \%$ 20\% of osteosarcoma patients are presented with high-grade subclinical metastatic disease that metastasizes at very early stages[1,2]. Although chemotherapy combined with surgery is the standard of care for osteosarcoma, the prognosis in patients with metastatic osteosarcoma remains poor; besides, $20-40 \%$ of patients still experience tumor recurrence or distant metastasis following this treatment[2].

TAM, a dominant population of infiltrating inflammatory cells in a majority of malignant tumors, plays an important role in tumor progression through the production of soluble mediators that possibly promotes proliferation, matrix remodeling, invasion, immune evasion, and chemoresistance. M2 macrophages are known to have strong tumor-supporting functions as they contribute to the establishment of a local 
immunosuppressive microenvironment[3-6]. Phenotypically and functionally, macrophages can be polarized in two main directions, activated pro-inflammatory M1 macrophages with antitumor properties and alternatively, activated anti-inflammatory M2 macrophages with tumor-supporting functions. The polarization of macrophages have been more recently demonstrated by Low et al.[7-10], wherein, the coculture of nasopharyngeal carcinoma (NPC) cells and macrophages exerted pro-tumoral functions by inducing various inflammatory cytokines during the early stage; however, up-regulated the induction of cancer-related genes in macrophages in the later stages, suggesting that recruited macrophages into the NPC tumor microenvironment was tuned to promote tumor progression. In the gastric cancer tumor microenvironment, crosstalk between tumor cells and TAMs induced the secretion of inflammatory factors including II-6, TNF- $a$, and CCL2, via the NF-KB signaling cascade to promote the development of gastric cancer[11-14]. Besides, the effect of TAMs on tumor progression possibly depends on the tumor type, TME, and the localization of TAMs. Given the increasing evidence supporting active crosstalk between TAMs and EMT in tumor metastasis and progression, investigating the signaling pathways involved in this process might provide an insight into new strategies for the treatment of invasive cancer including osteosarcoma.

Exosomes are lipid bilayer-enclosed nanovesicles with a size range of $40-200 \mathrm{~nm}$ that are secreted by nearly all cell types, including tumor cells, and are extensively found in all body fluids[15-17]. Exosomes enclose various types of molecules including lipids, proteins, DNA, messenger RNA (mRNA), and noncoding RNAs, microRNA and long non-coding RNA (IncRNA). Thus, exosomes may mediate specific intercellular communication, exchange of products with other cells, eliminate undesired products from cells, and activate signaling pathways in cells they fuse or interact with. Increasing evidence also suggested that exosomes may participate in the formation and progression of different cancer processes, including remodeling of the tumor microenvironment (TME), angiogenesis, immune escape, dissemination and metastasis[18]. A plethora of evidence indicates that tumor cell-derived exosomes could interact with immune cells in the TME to assist the immune escape of tumor cells and promote tumor progression and therapy resistance in cancer cells. Furthermore, exosomes rich in miR-21 secreted by head and neck tumors could induce M2 phenotype differentiation, thus promoting tumor progression and metastasis[19]. Recent studies have demonstrated that macrophages, particularly M2 macrophages, play an extremely crucial role in the occurrence and development of tumors by inducing immunosuppression, promoting tumor metastasis, and tumor drug resistance[20-24].

The T cell immunoglobulin and mucin domain (Tim) family proteins are type-l transmembrane proteins expressed by diverse cell types within the immune systems of rodents and humans. In 2001, proteins of the Tim family were initially identified in mice T lymphocytes (Tim-1, Tim-2, Tim-3, and Tim-4) and subsequently in humans ( $\mathrm{Tim}-1, \mathrm{Tim}-3$, and $\mathrm{Tim}-4)$. These proteins can regulate $\mathrm{T}$ cell activation, tolerance, and diverse effectors pathways of the immune response. Tim-3, also known as HAVCR2, was initially identified to be selectively expressed in the activated Th1 cells, its expression was later confirmed in diverse immune cell types, including regulatory T cells (Tregs), macrophages, dendritic cells, natural killer (NK) cells and in a variety of cells in different types of tumors[25,26]. Scientific evidence indicates that Tim-3 could influence the occurrence and development of diseases by regulating the function of 
macrophages in various diseases. Moreover, Tim-3 could promote immune tolerance by inhibiting the activation of macrophage-mediated by LPS/TLR through interacting with STAT1 in infectious diseases[27]. Tim-3 could induce macrophage M2 differentiation and aggravate podocyte damage to participate in the occurrence and development of the disease through NF-KB/TNF-a pathway in diabetic nephropathy $[28,29]$. In osteosarcoma, it has been documented that the expression of Tim-3 is significantly increased in tumor tissue[30], and the overexpression of Tim-3 could promote the occurrence and development of osteosarcoma. However, whether the overexpression of Tim-3 in osteosarcoma could also regulate the differentiation of tumor-associated macrophages and the underlying molecular mechanism of this regulation remains elusive. Therefore, this study was initiated to investigate the effect of osteosarcoma-derived exosomes on M2 macrophage polarization and to elucidate the underlying molecular mechanism in the occurrence and development of osteosarcoma. Furthermore, this study also provides a theoretical basis and experimental evidence for refining the therapeutical strategy and improving the prognosis of patients with osteosarcoma.

\section{Materials And Methods}

\section{Tissue samples}

Tumor tissue samples were obtained from 18 patients with primary osteosarcoma who received a histopathological diagnosis and underwent surgical resection for osteosarcoma at the department of orthopedics and pediatric surgery of our hospital from March 2018 to October 2019 were collected. We excluded patients with a prior diagnosis of tumors of other organs and patients with serious diseases of heart, lung and other organs. The tissue parts surrounding the tumor, which was not diagnosed as cancer tissue, from the same patient was collected as normal control. This study was approved by the Institutional Ethics Review Board of Huanggang Central Hospital. Written informed consent was obtained from all the patients. Tissue samples were collected at surgery, immediately frozen in liquid nitrogen and stored until analysis in the present study.

\section{Cell lines and main reagents}

Human osteosarcoma MG63 and normal osteoblast hFOB cell lines were purchased from the Cell Bank Type Culture Collection of the Chinese Academy of Sciences (Shanghai, China). Dulbecco's modified Eagle's medium-F12 (DMEM/F12) was purchased from Hyclone, USA; Fetal Bovine Serum (FBS), Penicillin-streptomycin $(100 \mathrm{U} / \mathrm{mL})$ were purchased from Hangzhou Sijiqing Biological Company; RIPA Lysis buffer, BCA Protein Quantification Reagent Kit were purchased from Invitrogen, USA; Opti-MEM (Gbico, UK); Anti-Tim-3 monoclonal antibody was obtained from BD Bioscience, USA; Rabbit anti-ECadherin, N-cadherin, Vimentin, and GAPDH monoclonal antibodies were purchased from Abcam, USA; Rabbit anti-CD9, CD81, and CD63 monoclonal antibodies were purchased from Santa Cruz, USA; Horseradish peroxidase (HRP) labeled goat anti-rabbit IgG secondary antibody was purchased from 
Guangzhou Jingcai; PKH67 green fluorescent cell linker dye, and PMA were purchased from Sigma Company, USA.

\section{Cell culture and transfection}

After resuscitation of osteosarcoma cell lines MG63 and hFOB, the cells were cultured in DMEM/F12 medium supplemented with $10 \% \mathrm{FBS}$ and $1 \%$ of penicillin-streptomycin at $37^{\circ} \mathrm{C}$ in a humidified incubator with $5 \% \mathrm{CO}_{2}$. When the cells reached $70 \%-80 \%$ confluence, the cells were digested with $0.25 \%$ trypsin and centrifuged at $800 \mathrm{r} / \mathrm{min}$ for $5 \mathrm{~min}$ at room temperature (RT). The supernatant was discarded, and the cells were resuspended with DMEM/F12 for passage.

The MG63 cells $\left(2 \times 10^{5}\right.$ cells/well) at the logarithmic growth stage were seeded onto a 6-well plate and incubated overnight. MG63 cells were transfected with $30 \mathrm{ng}$ of Tim-3 sgRNA, Cas 9 plasmid or negative control (NC) sequence (Guangzhou Ruibo Biological Company) using Transfection reagent Lipofectamine 3000 (Invitrogen, USA) following the manufacturer's instructions. After transfection for $6 \mathrm{~h}$, the medium was replaced with DMEM/F12 medium containing $10 \%$ FBS and incubated in a humidified atmosphere of $5 \% \mathrm{CO}_{2}$ at $37^{\circ} \mathrm{C} .48 \mathrm{~h}$ after transfection, cells were collected for the downstream experiments. The experiments were repeated three times. The required macrophages in the experiment were obtained by treating about $1 \times 10^{6} \mathrm{THP}-1$ cells with $50 \mathrm{ng} / \mathrm{mL}$ PMA for $48 \mathrm{~h}$.

\section{Isolation and identification of exosomes}

MG63 and hFOB cells at 80\% 90\% confluence were pelleted and washed thrice with DMEM/F12 media, followed by replacement of media with fresh DMEM/F12 containing 10\% FBS and the cells were cultured for additional $72 \mathrm{~h}$ at $37^{\circ} \mathrm{C}$ in a $5 \% \mathrm{CO}_{2}$ atmosphere before harvesting exosomes. The cell culture medium was collected and intact cells and cell debris were removed and exosomes were isolated by differential centrifugation at $300 \times \mathrm{g}$ for $10 \mathrm{~min}$, and 2,000×g for $10 \mathrm{~min}, 1,000 \times \mathrm{g}$ for $30 \mathrm{~min}$, respectively. The resulting pellet was resuspended, washed once in ice-cold PBS, and filtered through sterile $0.22 \mu \mathrm{m}$ (pore-size) syringe filter and repelleted at $1,000,000 \mathrm{~g}$ for $70 \mathrm{~min}$ at $4{ }^{\circ} \mathrm{C}$. The pellet containing exosomes was washed and resuspended in ice-cold PBS and stored at $-80^{\circ} \mathrm{C}$.

For Transmission Electron Microscopy (TEM), freshly prepared osteosarcoma-exosomes (2 LL) were diluted in 20 L PBS, fixed with 3.5\% paraformaldehyde, then, exosome preparation was allowed to adsorb in the mesh copper grid. The resulting grids were rinsed twice with wash buffer and contrasted by uranyl-oxalate solution at $\mathrm{pH} 7$ for $5 \mathrm{~min}$. The TEM visualizations of exosomes morphology were performed using a Hitachi $\mathrm{H}-9000$ transmission electron microscope at $300 \mathrm{kV}$ and images were acquired using a slow-scan CCD camera. Size profiles of isolated exosomes were determined by the Nanosight size analyzer. Briefly, $50 \mathrm{LL}$ of exosomes was diluted in PBS to a final volume of $1 \mathrm{ml}$ and filtered through a $0.22 \mu \mathrm{m}$ filter membrane. Size curves were determined by nanoparticle tracking analysis using a Nanosight NS300TM. Three video recordings of $40 \mathrm{sec}$ were carried out for each preparation. 
NTA 3.2 software version was used to record and analyze the videos. The expression of exosome characteristic proteins including CD9, CD81, and CD63 was detected using Western blot assay.

\section{Labeling and tracking of exosomes}

The extracted and purified exosomes were collected and labeled KPH67 reagent according to the manufacturer's instructions. The nuclei of macrophages were counter-stained with DAPI dye following the manufacturer's instructions. KPH67 labeled exosomes were added to DAPI stained macrophages and cocultured at $37{ }^{\circ} \mathrm{C}$ in a humidified atmosphere of $5 \% \mathrm{CO}_{2}$ for $12 \mathrm{~h}$. After incubation, the exosomes were analyzed by a fluorescence confocal microscope.

\section{Cell migration and invasion}

The cell invasion assay was carried out using 24-well transwell chambers with $6.5 \mathrm{~mm}$ diameter polycarbonate filters ( $8 \mu \mathrm{m}$ pore size, Corning, NY, USA) coated with $35 \mu \mathrm{L}$ Matrigel (Becton, Dickinson and Company, USA). Cells were seeded in the upper chambers at a density of $5 \times 104$ cells/well and then treated with different concentrations of Anlotinib $(0,2,4,8 \mu \mathrm{mol} / \mathrm{L})$, and allowed to invade for $24 \mathrm{~h}$ toward the lower chamber containing $10 \%$ FBS containing $10 \%$ FBS or serum-free media. After washing twice with PBS, cells invading through the Matrigel layer were fixed with methanol followed by mounting and staining with Giemsa stain for $15 \mathrm{~min}$. The inner side of the chambers was wiped with a cotton swab. The number of invading cells from at least five fields of each of three separate membranes was counted under an inverted microscope (Olympus Cor., Tokyo, Japan). Three independent experiments were carried out in triplicate.

The MG63 cells were routinely digested and resuspended in DMEM/F12 medium without FBS and seeded at a density of $5 \times 10^{4}$ cells/well in the upper chamber of Transwell containing $200 \mathrm{LL}$ serum-free medium allowed to invade for $24 \mathrm{~h}$ toward the lower chamber containing $1 \times 10^{4}$ cells $/ \mathrm{mL}$ macrophages and 800 $\mathrm{GL}$ of conventional DMEM/F12 medium supplemented with $10 \%$ FBS. After washing twice with PBS, cells invading through the Matrigel layer were fixed with absolute alcohol for $15 \mathrm{~min}$, dried at RT, followed by mounting and staining with $0.1 \%$ crystal violet for $15 \mathrm{~min}$ at RT. The inner side of the chambers was wiped with a cotton swab. The number of invading cells from at least five fields of each of three separate membranes was counted under an inverted microscope (Olympus Cor., Tokyo, Japan). Three independent experiments were carried out in triplicate.

\section{Quantitative Real-time PCR (qRT-PCR)}

The total RNA was extracted from exosomes or MG63, hFOB cells, and transfected MG63 cells extracted using Trizol Reagent (Invitrogen). The RNA quality and concentration were measured using a spectrophotometer. The reverse transcription reaction was performed to reverse transcribe RNA into cDNA 
using RevertAidTM First Strand cDNA Synthesis Kit (Thermo Scientific) following the manufacturer's protocols. qRT-PCR was performed with the SYBR Green Master Mix Kit (Shanghai Solarbio Biological Company) on an ABI Prism 7000 sequence detection system (Applied Biosystems). Expression of target genes was normalized to the expression of GAPDH relative gene expression levels were quantified using the 2- $\Delta \Delta$ Ct method. Three independent experiments were performed in triplicates. The following primers were synthesized (Shanghai Biotech) and used for RT-PCR: Tim-3-F: 5'-

ACTCTACTCTACCTACATCTGGGACACT-3', Tim-3-R: 5'-GTAGGTCCCATGGTCATCCAG-3'; Arg-1-F: 5'TTGGGTGGATGCTCACACTG-3', Arg-1-R:5'-TTGCCCATGCAGATTCCC-3';CD206-F:5'-

CAGGTGTGGGCTCAGGTAGT -3', CD206-R:5'-TGTGGTGAGCTGAAAGGTGA-3; CD163-F:5'GCCGGCTATCCAGACAATTA-3', CD163-R:5'-GGCCAAACTGAGGTGGTTTA-3'; iNOS-F:5'CAGCTGGGCTGTACAAACC TT-3';iNOS-R:5'-CAGCTGGGCTGTACAAACCTT-3'; IL-10-F:5'TGCTATGCTGCCTGCTCTTA-3', IL-10-R:5'-TCATTTCCGATAAGGCTTGG-3'; GAPD-F: 5'GGAGAAACCTGCCAA GTATG-3', GAPD-R:5'-TTACTCCTTGGAGGCCATGTAG-3'; TGF- $\beta$-F5'GGGATGAGCTAGTGCTGATCTGG-3', TGF- $\beta$-R:5'-AAACTTGCTCCATGTCCTGCTCTA-3'; VEGF-F:5'TGCAGGTGATGCTGACAGAGG-3', VEGF-R:5'- GGATGAGCTAGTGCTGATCTGG-3'

\section{Western blot Assay}

Total proteins were extracted from the osteosarcoma and para-carcinoma tissues or exosomes or MG63, hFOB cells or transfected MG63 cells on ice with radio-immunoprecipitation assay (RIPA) cell lysis buffer supplemented with protease inhibitors. Protein concentrations were quantified by the BCA method. Cell lysates were separated using $12 \%$ sodium dodecyl sulfate-polyacrylamide gel electrophoresis (SDSPAGE) and then transferred the proteins onto the polyvinylidene difluoride (PVDF) membrane. The membranes were blocked with 5\% skim milk in Tris-buffered saline with Tween-20 for $2 \mathrm{~h}$ at RT. Subsequently, the membrane was incubated with primary antibodies including rabbit anti-mouse/human Tim-3 (1: 500), E-cadherin (1: 800), N-cadherin (1: 800), Vimentin (1: 800), and CD9 (1: 600), CD81 (1: 600), CD63 (1: 600), GAPDH (1: 1000) in 5\% blocking buffer overnight at $4{ }^{\circ} \mathrm{C}$. Then, the membranes were washed twice with TBST solution for $5 \mathrm{~min} /$ time, incubated with horseradish enzyme-labeled secondary antibody (1: 5000) for $1 \mathrm{~h}$ at RT. The target bands were visualized using the Enhanced Chemiluminescence Kit and the protein bands were quantified using Image $\mathrm{J}$ software. Using GAPDH as the internal reference, the ratio of gray value between the target band and internal reference band was considered as the relative expression of the target proteins. Three independent experiments were performed in triplicates.

\section{Pulmonary metastases of osteosarcoma}

The animals were cared for in accordance with the Guide for the care and use of laboratory animals in China. All procedures and animal experiments were approved by the Animal Protection and application committee of Huanggang Central Hospital. Fifteen male BALB/c mice (age 4-5 weeks, weight 200 to 250 
g) were obtained from the Institute of Zoology, Chinese Academy of Sciences. Mice were housed under specific-pathogen-free (SPF) controlled conditions of $(22 \pm 1)^{\circ} \mathrm{C}$ temperature, $60 \% \pm 10$ humidity with a 12 $\mathrm{h}$ light/dark cycle and ad libitum access to water and sterile food pellets Mice were randomly divided into 3 groups (5 in each group). MG63 and macrophages induced by MG63-Exo or NC-Exo or Tim-3 KO-Exo were administered via tail vein to observe the lung metastasis of osteosarcoma. After 5 weeks, mice in each group were anesthetized and sacrificed. All the suspected lesions of lung metastasis collected and fixed with $10 \%$ buffered formalin for (12-24) $h$ at RT, then paraffin-embedded, and sectioned into $4-\mu \mathrm{m}$ thick sections. The sections were prepared for histologic examination following staining with hematoxylin and eosin (H\&E).

\section{Statistical analysis}

Statistical analyses were performed using the SPSS software package (version 19.0; IBM, Chicago, IL, USA). All graphs were produced by GraphPad Prism 5.0 for Windows software (GraphPad Software Inc., La Jolla, CA, USA). Data were expressed as mean \pm standard deviation (SD). The comparison between the two groups was performed using the independent sample $t$ test, and the comparison among multiple groups was performed by one-way analysis of variance (ANOVA) followed by Bonferroni's correction post hoc test for multiple comparisons. All tests were 2 -sided, and $P<0.05$ was considered statistically significant.

\section{Results}

\section{MG63-Exo promoted the polarization of induced- macrophages into M2 phenotype}

Exosomes, explicitly hFOB-Exo and MG63-Exo, were isolated from the cell culture supernatant of normal osteoblast hFOB and osteosarcoma cell line MG63 by ultracentrifugation. TEM image analysis revealed that the morphology of the exosomes was round or oval (Figure 1A). The size of exosomes was ranged from about 30 to $150 \mathrm{~nm}$ based on size analysis (Figure 1B). Western blot assay results indicated that exosome-specific molecular marker including CD9, D81, and CD63 was significantly highly expressed in hFOB-Exo and MG63-Exo (Figure 1C). Macrophages were the most abundantly expressed immune cells in the TME. Furthermore, the effect of osteosarcoma-derived exosomes on macrophage differentiation was also investigated. Human monocyte line THP-1 was differentiated into macrophages under PMA stimulation. As illustrated in Figure 1D, the suspension of THP-1 monocytes could be differentiated into adherent-growing macrophages and extended pseudopodia from the cell body under an optical microscope. Further, qRT-PCR results revealed that the expression of macrophage marker CD68 in THP-1 was significantly over-expressed following stimulation with PMA in comparison with the control group (Figure 1E). In order to confirm that the isolated exosomes could be phagocytized by macrophages, we labeled the exosomes with the green fluorescent dye PKH67 beforehand, which was co-cultured with macrophages for $12 \mathrm{~h}$. Notably, the green fluorescence around the nuclei of macrophages was observed 
under confocal fluorescence microscopy, indicating that exosomes were phagocytized by macrophages (Figure 1F). qRT-PCR data suggested that compared with the PBS control group or macrophages cocultured with hFOB-Exo, MG63-Exo significantly promoted the expression of M2-type macrophage markers CD206, CD163, and Arginase-1; however, no alterations in the expression of iNOS, the marker of M1 macrophage, was noted. Conceivably, hFOB-Exo exhibited no apparent effect on the M1 or M2 phenotype differentiation of macrophages (Figure 1G). We further applied qRT-PCR to detect the effect of macrophages on the expression levels of M2 type cytokines, TGF- $\beta, \mathrm{IL}-10$ and VEGF under different treatment conditions. The results indicated that compared with the control group, MG63-Exo significantly promoted the expression of TGF- $\beta$, IL-10 and VEGF; however, hFOB-Exo did not affect macrophages for the expression of the above cytokines (Figure $1 \mathrm{H}$ ).

\section{M2 macrophages induced by MG63-Exo promoted the migration, invasion, and EMT of osteosarcoma cells}

To clarify the effects of M2 macrophages induced by MG63-Exo on the migration, invasion, and EMT of osteosarcoma cell in vitro, we used a co-culture system for the detection (Figure 2A). The results of migration and invasion assays revealed that compared with the control groupa, M2 macrophges induced by MG63-Exo could significantly promote the migration and invasion ability of osteosarcoma cells (Figure 2B). Furthermore, we investigated the effect of M2 macrophages induced by MG63-Exo on EMT of tumor cells. Western blot assays results indicated that as compared with the control group, M2 macrophages induced by MG63-Exo significantly reduced the expression of the epithelial cell marker Ecadherin in osteosarcoma cells and remarkably promoted the expression of mesenchymal cell markers $\mathrm{N}$ cadherin and Vimentin (Figure 2C).

\section{Overexpression of Tim-3 protein in MG63-Exo could be transferred to macrophages by exosomes}

Previous studies have revealed that exosomes contained a large number of bioactive maternal cell proteins that could be passed on into other cells to affect their cell function. We analyzed the expression of Tim-3 in osteosarcoma and para-carcinoma tissues of 18 patients by Western blot assay. The results revealed that the positive rate of Tim-3 expression in osteosarcoma was significantly higher than that in para-carcinoma tissue (Figure 3A, B). To further clarify whether MG63-Exo could overexpress Tim-3, we used qRT-PCR and Western blot assays to detect the expression of Tim-3 mRNA and protein in MG63 cells. The results showed that compared with normal osteoblast hFOB cells, the expression of Tim-3 mRNA and protein in MG63 cells was significantly up-regulated (Figure 3C, D). Using Western blot assay, we detected the protein expression Tim-3 protein in exosomes. Furthermore, as compared with human normal osteoblast hFOB-Exo, the expression of Tim-3 protein in MG63-Exo was also significantly upregulated (Figure 3E). To further investigate whether MG63-Exo could deliver Tim-3 into macrophages, as presented in Figure F, we found that the expression of Tim-3 protein in macrophages co-cultured with 
MG63-Exo was significantly higher than that in macrophages co-cultured with hFOB-Exo or PBS. Furthermore, we knocked down the expression of Tim-3 (Tim-3 KO) in MG63 cells by CRISPR/Cas9. qRT-PCR and Western blot results indicated that compared with the cells in the NC group, the expression of Tim-3 mRNA and protein in MG63 cells in the Tim-3 KO group were significantly inhibited (Figure 3G, $\mathrm{H}$ ). The identification and characterization of MG63 exosomes with Tim-3 knockout (Tim-3 KO-ExO) was carried out by particle size analysis and Western blot analysis (Figure $3 \mathrm{l}, \mathrm{J}$ ). We also detected the protein expression of Tim-3 in Tim-3 KO-Exo by Western blot assay. These results suggested that compared with MG63-Exo, the expression of Tim-3 in Tim-3 KO-Exo was significantly down-regulated (Figure 3K). At the same time, we continued to adopt Western blot assay to detect the expression of Tim3 protein in macrophages co-cultured with Tim-3 KO-ExO, the findings indicated that the expression of Tim-3 was significantly decreased in macrophages co-cultured with Tim-3 KO-Exo compared with macrophages co-cultured with NC-Exo or MG63-Exo (Figure 3L). Collectively, these results indicated that overexpression of Tim-3 protein in MG63-Exo could be transferred into macrophages by exosomes; however, the down-regulation of Tim-3 expression in MG63 cells reduced the expression of Tim-3 in cocultured macrophages by down-regulating the expression of Tim-3 in MG63-Exo.

\section{Tim-3 derived from MG63-Exo promoted the migration, invasion, and EMT of osteosarcoma cells by promoting $\mathrm{M} 2$ macrophage polarization}

The above experimental results indicated that MG63-Exo induced the polarization of macrophages into the M2 phenotype to promote the migration, invasion, and EMT of osteosarcoma cells, while Tim-3, which was highly expressed in MG63-Exo, could be phagocytized by macrophages. Thus, we speculated that Tim-3 might play a key role in supporting this process. Tim-3 was secreted by MG63-Exo and internalized by macrophages, which could promote the metastasis, invasion, and EMT of osteosarcoma cells by promoting M2 macrophage polarization. To confirm this hypothesis, we first identified the effect of Tim-3 KO-Exo on the expression of M2-type macrophage markers CD206, CD163, arg-1, and the cytokines TGF- $\beta$, IL-10, and VEGF. The results indicated that compared with macrophages co-cultured with NC-Exo or MG63-Exo, the expression of M2 macrophage marker including CD206, CD163, Arginase1 , cytokines TGF- $\beta$ and cytokines IL-10, VEGF were significantly decreased following treatment with Tim-3 KO-Exo (Figure 4A, B). The results of migration and invasion experiments revealed that compared with macrophages induced by NC-Exo or MG63-Exo co-cultured with MG63, the macrophages induced by Tim-3 KO-Exo could significantly inhibit the migration and invasion ability of MG63 cells (Figure 4C). EMT findings further also confirmed that compared with macrophages induced by NC-Exo or MG63-Exo co-cultured with MG63, macrophages induced by Tim-3 KO-Exo significantly promoted the expression of the epithelial cell marker E-cadherin and inhibited the expression of mesenchymal cell markers $\mathrm{N}$-cadherin and Vimentin (Figure 4D). The findings indicated that MG63-Exo induced macrophages to differentiate into the M2 phenotype through Tim-3, which could further promote the metastasis, invasion, and EMT of 
osteosarcoma cells MG63; however, the down-regulation of Tim-3 expression in MG63-Exo could reverse the above-mentioned effects of MG63-Exo.

\subsection{Tim-3 derived from MG63-Exo promoted lung metastasis of osteosarcoma cells in nude mice by promoting M2 phenotype macrophage polarization}

Next, to further evaluate the role of Tim-3 derived from exosome on the occurrence and development of osteosarcoma cells in vivo after activating macrophages to differentiate into M2-type, we administered macrophages induced with Tim-3 Ko-Exo or NC-Exo and osteosarcoma MG63 cells into nude mice via the tail vein. After 5 weeks, the results indicated that compared with the nude mice treated with MG63 cells and macrophages induced by Tim-3 Ko-Exo, larger and multiple metastatic tumor nodules were deposited in the lung of nude mice treated with MG63 cells and macrophages induced by NC-Exo or MG63-Exo (Figure 5). These findings indicated that Tim-3 in MG63-Exo could promote lung metastasis of osteosarcoma in vivo by promoting M2 macrophage polarization.

\section{Discussion}

Exosomes, as molecular information carriers, play a potent role in the mediation of cell-to-cell communication between tumor cells and immune cells to induce a pro-tumoral environment and to eventually promote the tumor progression and survival[31,32]. A plethora of evidence also indicated that exosomes derived from tumors were crucially involved in the alteration of the biological activities of their recipient cells through the transfer of their oncogenic content. TAM, as the major type of immune cell in tumor tissues, well-recognized to play a role in the occurrence, invasion, and metastasis of tumor cells[33-36]. Furthermore, accumulating studies have indicated the tumor-supporting functions of TAMs for many types of malignancies, including breast cancer, lung adenocarcinoma, esophageal cancer, cervical cancer, ovarian cancer, prostate cancer, and renal cell carcinoma. Moreover, mature macrophages could be reprogrammed into different types under different environmental conditions, such as M1 and M2, which plays differential roles in tumor tissue. In gastric cancer, studies have demonstrated that proinflammatory M1-type macrophages could promote apoptosis and inhibit the growth of gastric cancer cells, indicating the antitumor properties of macrophages. However, activated anti-inflammatory M2-type macrophages with tumor-supporting functions could promote the growth and invasion of tumor cells[37]. In liver cancer, M2-type macrophages could promote the malignant transformation and progression of liver cancer, through the induction of expression of vascular endothelial factors and matrix metalloproteins in the TME in comparison with M1-type macrophages, and facilitate the invasion and metastasis of liver cancer, which are important factors for poor prognosis of patients with liver cancer[33]. The tumor-supporting functions of TAMs have been demonstrated for in many types of malignancies including ovarian cancer, nasopharyngeal cancer, and melanoma, TAM was dominated mostly by M2-type macrophages; however, the reprogramming of M2 macrophages towards M1 
phenotype exhibited a marked negative impact on tumor progression and prognosis[7,38,39]. These pieces of evidence thus indicated that M1-type macrophages present an inhibitory effect on tumor immunity, while M2-type macrophages exhibit a tumor-supporting function including tumor invasion and metastasis. In this study, we isolated the exosomes from the culture supernatant of the osteosarcoma cell line MG63 and further investigated the effect overexpression of Tim-3 mediated by exosomes on the migration and invasion of osteosarcoma cells. Using TEM, morphology and size profiles of the isolated exosomes were determined and the results indicated that the obtained exosomes were round or oval in structure with a size ranging from 30 to $150 \mathrm{~nm}$. The Western blot assay identified a high expression of exosomes related marker such as CD9, CD63, and CD81 Studies have demonstrated that breast cancerderived exosomes activated NF-KB signaling pathway through TLR2 to induce macrophage activation, while the activated macrophages regulated immunity through secretion of inflammatory factors such as IL-6/TNF-a, GCSF and CCL2 to promote distant metastasis of breast cancer[40]. Furthermore, in colon cancer, tumor-derived exosomes mediated the M2 polarization of TAM by IncRNA PPH1 to facilitate the metastasis and invasion of colon cancer cells[41]. Thus, to further clarify the effect of osteosarcomaderived exosomes on macrophage differentiation, migration, invasion, and EMT of osteosarcoma cells, we induced THP- 1 cells to polarize mature macrophages by PMA and identified the expression of macrophage marker protein CD68 by Western blot assay. Subsequently, the effect of osteosarcomaderived exosomes on the differentiation of macrophages was evaluated by qRT-PCR. The experimental results indicated that exosomes derived from osteosarcoma could significantly promote the $\mathrm{M} 2$ differentiation of macrophages and secretion of cytokines IL-10, TGF $\beta$, and VEGF. Further, the Transwell co-culture assay revealed that exosomes induced macrophages significantly promoted the migration and invasion ability of osteosarcoma cells. Taken together, these systematic assays concluded that the isolated osteosarcoma-derived exosomes contained crucial factors, which were related to cellular invasion, development and cell proliferation. Tim-3, a negative regulator of immunity, is selectively induced upon the differentiation of naïve Th cells into interferon-gamma secreting Th1 effector cells. Tim-3 by binding to its ligand Galectin-9 (Gal-9) could induce apoptosis and immune tolerance of T cells. In this study, Tim-3 was also found to be expressed in macrophages, NK cells, and other natural immune cells, which may play an important role in maintaining immune homeostasis[30]. Using Western blot assay, we detected high expression of Tim-3 protein in osteosarcoma tissues and cells., which was consistent with previous studies. Further, to clarify that the overexpressed Tim-3 in osteosarcoma cells were phagocytized by macrophages through exosomes and induced the oncogenic reprogramming of macrophages into M2 type, we knocked down Tim-3 expression in osteosarcoma cells by CRISPR/Cas 9 and detected the expression of Tim-3 in osteosarcoma-derived exosomes derived by Western blot analysis. The results indicated that the knocked down of Tim-3 in osteosarcoma cells could significantly down-regulated the expression of Tim-3 in exosomes, while the latter displayed no significant effect on M2 type differentiation of macrophages as well as on the expression of IL-10, TGF $\beta$ and VEGF. Further, Transwell and Western blot assays also demonstrated that under-expression of Tim-3 in exosomes exhibited no apparent promoting effect on the migration, invasion, and EMT of osteosarcoma cells. The distant metastasis of osteosarcoma in nude mice further confirmed that Tim-3 expression in exosomes 
promoted the lung metastasis of osteosarcoma cells by inducing macrophage differentiation; however, the down-regulation of Tim-3 expression in exosomes significantly reduced metastasis in the lung.

\section{Conclusions}

This study identified that osteosarcoma-derived exosomes induced M2 type polarization of macrophages through regulation of the expression of Tim-3. Furthermore, the differentiated macrophages promoted the migration, invasion, EMT, and distant metastasis ability of osteosarcoma cells through the secretion of IL-10, TGF $\beta$, VEGF. However, the role of exosomes in tumor microenvironment remains complex and variable and therefore the above-mentioned mechanism may represent a probable mechanism and warrant further investigations.

\section{List Of Abbreviations}

TAM: tumor-associated macrophage; NPC: nasopharyngeal carcinoma; mRNA: messenger RNA; IncRNA: long non-coding RNA; TME: tumor microenvironment; Tim: T cell immunoglobulin and mucin domain; Tregs: regulatory $\mathrm{T}$ cells.

\section{Declarations}

\section{Ethics approval and consent to participate}

This study was approved by the Institutional Ethics Review Board of Huanggang Central Hospital. Written informed consent was obtained from all the patients. Tissue samples were collected at surgery, immediately frozen in liquid nitrogen and stored until analysis in the present study.

\section{Availability of data and materials}

All data are available in the included figures and tables.

\section{Competing interests}

The authors declare that they have no conflict of interests.

\section{Acknowledgements}

None.

\section{Consent for publication}


None.

\section{Funding}

This work is supported by Huanggang Science and Technology and Development Plan Project(s201501-0130084) and Scientific Research Project of Hubei Provincial Health and Family Planning Commission Medical College (WJ2016-Y-42).

\section{Authors' contributions}

Conceptualization: ZC, WX; Methodology: ZC, LW, CW; Software: CW, LH, YR; Supervision: WX; Writing original draft: ZC, LW; All authors read and approved the final manuscript.

\section{References}

1. Schiavone K, Garnier D, Heymann M-F, Heymann D, The heterogeneity of osteosarcoma: the role played by cancer stem cells, in Stem Cells Heterogeneity in Cancer. 2019, Springer.1139, 187-200.

2. Pollino S, Palmerini E, Dozza B, Bientinesi E, Piccinni-Leopardi M, Lucarelli E, Righi A, Benassi MS, Pazzaglia L. CXCR4 in human osteosarcoma malignant progression. The response of osteosarcoma cell lines to the fully human CXCR4 antibody MDX1338. Journal of bone oncology 2019; 17: 100239.https://doi.org/10.1016/j.jbo.2019.100239

3. Hung C-H, Chen F-M, Lin Y-C, Tsai M-L, Wang S-L, Chen Y-C, Chen Y-T, Hou M-F. Altered monocyte differentiation and macrophage polarization patterns in patients with breast cancer. BMC cancer 2018; 18(1): 366.https://doi.org/10.1186/s12885-018-4284-y

4. Saito A, Ohzawa H, Tojo M, Kumagai Y, Kanamaru R, Tsukui H, Shiba S, Ito H, Sata N, Kitayama J, Metformin inhibits monocyte differentiation to tumor associated macrophage (TAM) and may restore anti-tumor immunity. 2019, AACR.

5. Guc E, DeMarco GS, Pieracci G, Pollard JW, Tenascin C affects tumor associated macrophage phenotype and tumor invasion in breast cancer. 2019, AACR.

6. Gionfriddo G, Plastina P, Augimeri G, Catalano S, Giordano C, Barone I, Morelli C, Giordano F, Gelsomino L, Sisci D. Modulating Tumor-Associated Macrophage Polarization by Synthetic and Natural PPARy Ligands as a Potential Target in Breast Cancer. Cells 2020; 9(1):

174.https://doi.org/10.3390/cells9010174

7. Low HB, Png CW, Li C, Wang DY, Wong SBJ, Zhang Y. Monocyte-derived factors including PLA2G7 induced by macrophage-nasopharyngeal carcinoma cell interaction promote tumor cell invasiveness. Oncotarget 2016; 7(34): 55473.https://doi.org/10.18632/oncotarget.10980

8. Li MZ, Wu YH, Ali M, Wu XQ, Nie MF. Endometrial stromal cells treated by tumor necrosis factor-a stimulate macrophages polarized toward M2 via interleukin- 6 and monocyte chemoattractant 
protein-1. Journal of Obstetrics and Gynaecology Research 2020.https://doi.org/10.1111/jog.14135

9. Lai YS, Wahyuningtyas R, Aui SP, Chang KT. Autocrine VEGF signalling on M2 macrophages regulates PD-L1 expression for immunomodulation of T cells. Journal of cellular and molecular medicine 2019; 23(2): 1257-1267.https://doi.org/10.1111/jcmm.14027

10. Chan M, Gómez-Aristizábal A, Gandhi R, Marshall W, Mahomed N, Viswanathan S. Ex vivo polarized pro-inflammatory vs. homeostatic monocytes/macrophages elicit differential responses within a human osteoarthritic joint explant model. Osteoarthritis and Cartilage 2019; 27: S379S380.https://doi.org/10.1016/j.joca.2019.02.378

11. Wu L, Zhang X, Zhang B, Shi H, Yuan X, Sun Y, Pan Z, Qian H, Xu W. Exosomes derived from gastric cancer cells activate NF-KB pathway in macrophages to promote cancer progression. Tumor Biology 2016; 37(9): 12169-12180.https://doi.org/10.1007/s13277-016-5071-5

12. Liu Y, Li L, Li Y, Zhao X. Research Progress on Tumor-Associated Macrophages and Inflammation in Cervical Cancer. BioMed Research International 2020; 2020.https://doi.org/10.1155/2020/6842963

13. Yadav MK, Inoue Y, Nakane-Otani A, Tsunakawa Y, Jeon H, Samir O, Teramoto A, Kulathunga K, Kusakabe M, Nakamura M. Transcription factor MafB is a marker of tumor-associated macrophages in both mouse and humans. Biochemical and biophysical research communications 2020; 521(3): 590-595.https://doi.org/10.1016/j.bbrc.2019.10.125

14. Yin M, Shen J, Yu S, Fei J, Zhu X, Zhao J, Zhai L, Sadhukhan A, Zhou J. Tumor-Associated Macrophages (TAMs): A Critical Activator In Ovarian Cancer Metastasis. OncoTargets and therapy 2019; 12: 8687.https://doi.org/10.2147/OTT.S216355

15. Xie C, Du L-Y, Guo F, Li X, Cheng B. Exosomes derived from microRNA-101-3p-overexpressing human bone marrow mesenchymal stem cells suppress oral cancer cell proliferation, invasion, and migration. Molecular and cellular biochemistry 2019; 458(1-2): 1126.https://doi.org/10.1007/s11010-019-03526-7

16. Exosomes E, Verweij FJ, Revenu C, Arras G, Bene FD, Niel GV, Verweij FJ, Revenu C, Arras G, Dingli F. Live Tracking of Inter-organ Communication by Resource Live Tracking of Inter-organ Communication by Endogenous Exosomes In Vivo. 2019.https://doi.org/10.1158/0008-5472

17. Wee I, Syn N, Sethi G, Goh BC, Wang L. Role of tumor-derived exosomes in cancer metastasis. Biochimica et Biophysica Acta (BBA)-Reviews on Cancer 2019; 1871(1): 1219.https://doi.org/10.1016/j.bbcan.2018.10.004

18. Kumar D, Gupta D, Shankar S, Srivastava RK. Biomolecular characterization of exosomes released from cancer stem cells: possible implications for biomarker and treatment of cancer. Oncotarget 2015; 6(5): 3280.https://doi.org/10.18632/oncotarget.2462

19. Hsieh C-H, Tai S-K, Yang M-H. Snail-overexpressing cancer cells promote M2-like polarization of tumor-associated macrophages by delivering MiR-21-abundant exosomes. Neoplasia 2018; 20(8): 775-788.https://doi.org/doi.org/10.1016/j.neo.2018.06.004

20. Biswas S, Mandal G, Chowdhury SR, Purohit S, Payne KK, Anadon C, Gupta A, Swanson P, Yu X, Conejo-Garcia JR. Exosomes Produced by Mesenchymal Stem Cells Drive Differentiation of Myeloid 
Cells into Immunosuppressive M2-Polarized Macrophages in Breast Cancer. The Journal of Immunology 2019; 203(12): 3447-3460.https://doi.org/10.4049/jimmunol.1900692

21. Guo Z, Song J, Hao J, Zhao H, Du X, Li E, Kuang Y, Yang F, Wang W, Deng J. M2 macrophages promote NSCLC metastasis by upregulating CRYAB. Cell death \& disease 2019; 10(6): 111.https://doi.org/10.1038/s41419-019-1618-x

22. Huang T, Song $C$, Zheng L, Xia L, Li Y, Zhou Y. The roles of extracellular vesicles in gastric cancer development, microenvironment, anti-cancer drug resistance, and therapy. Molecular cancer 2019; 18(1): 62.https://doi.org/10.1186/s12943-019-0967-5

23. Xu X, Ye J, Huang C, Yan Y, Li J. M2 macrophage-derived IL6 mediates resistance of breast cancer cells to hedgehog inhibition. Toxicology and applied pharmacology 2019; 364: 7782.https://doi.org/10.1016/j.taap.2018.12.013

24. Fortunato O, Borzi C, Milione M, Centonze G, Conte D, Boeri M, Verri C, Moro M, Facchinetti F, Andriani F. Circulating mir-320a promotes immunosuppressive macrophages M2 phenotype associated with lung cancer risk. International journal of cancer 2019; 144(11): 27462761.https://doi.org/10.1002/ijc.31988

25. Wing JB, Tay C, Sakaguchi S, Control of Regulatory T Cells by Co-signal Molecules, in Co-signal Molecules in T Cell Activation. 2019, Springer, 179-210.

26. Li X, Lu H, Gu Y, Zhang X, Zhang G, Shi T, Chen W. Tim-3 suppresses the killing effect of VY9Vס2 T cells on colon cancer cells by reducing perforin and granzyme B expression. Experimental cell research 2020; 386(1): 111719.https://doi.org/10.1016/j.yexcr.2019.111719

27. Motamedi M, Shahbaz S, Fu L, Dunsmore G, Xu L, Harrington R, Houston S, Elahi S. Galectin-9 Expression Defines a Subpopulation of NK Cells with Impaired Cytotoxic Effector Molecules but Enhanced IFN-y Production, Dichotomous to TIGIT, in HIV-1 Infection. ImmunoHorizons 2019; 3(11): 531-546.https://doi.org/10.4049/immunohorizons.1900087

28. Yang H, Xie T, Li D, Du X, Wang T, Li C, Song X, Xu L, Yi F, Liang X. Tim-3 aggravates podocyte injury in diabetic nephropathy by promoting macrophage activation via the NF-KB/TNF-a pathway. Molecular metabolism 2019; 23: 24-36.https://doi.org/10.1016/j.molmet.2019.02.007

29. Lin Y, Xu J, Lan H. Tumor-associated macrophages in tumor metastasis: biological roles and clinical therapeutic applications. Journal of hematology \& oncology 2019; 12(1):

76.https://doi.org/10.1186/s13045-019-0760-3

30. Pu F, Chen F, Zhang Z, Qing X, Lin H, Zhao L, Xia P, Shao Z. TIM3 expression and its association with overall survival in primary osteosarcoma. Oncology letters 2019; 18(5): 52945300.https://doi.org/10.3892/ol.2019.10855

31. Xue X, Zhao Y, Wang X, Qin L, Hu R. Development and validation of serum exosomal microRNAs as diagnostic and prognostic biomarkers for hepatocellular carcinoma. Journal of cellular biochemistry 2019; 120(1): 135-142.https://doi.org/10.1002/jcb.27165

32. Roman-Canal B, Moiola CP, Gatius S, Bonnin S, Ruiz-Miró M, González E, González-Tallada X, Llordella I, Hernández I, Porcel JM. EV-associated miRNAs from peritoneal lavage are a source of 
biomarkers in endometrial cancer. Cancers 2019; 11(6):

839.https://doi.org/10.3390/cancers11060839

33. Cheng L, Liu J, Liu Q, Liu Y, Fan L, Wang F, Yu H, Li Y, Bu L, Li X. Exosomes from melatonin treated hepatocellularcarcinoma cells alter the immunosupression status through STAT3 pathway in macrophages. International journal of biological sciences 2017; 13(6):

723.https://doi.org/10.7150/ijbs.19642

34. Zhang W, Zhang Y, He Y, Wang X, Fang Q. Lipopolysaccharide mediates time-dependent macrophage $\mathrm{M} 1 / \mathrm{M} 2$ polarization through the Tim-3/Galectin-9 signalling pathway. Experimental cell research 2019; 376(2): 124-132.https://doi.org/10.1016/j.yexcr.2019.02.007

35. Sanchez LR, Borriello L, Entenberg D, Condeelis JS, Oktay MH, Karagiannis GS. The emerging roles of macrophages in cancer metastasis and response to chemotherapy. Journal of leukocyte biology 2019; 106(2): 259-274.https://doi.org/10.1002/JLB.MR0218-056RR

36. Seoane S, Martinez-Ordoñez A, Eiro N, Cabezas-Sainz P, Garcia-Caballero L, Gonzalez LO, Macia M, Sanchez L, Vizoso F, Perez-Fernandez R. POU1F1 transcription factor promotes breast cancer metastasis via recruitment and polarization of macrophages. The Journal of pathology 2019; 249(3): 381-394.https://doi.org/10.1002/path.5324

37. Xu J, Yu Y, He X, Niu N, Li X, Zhang R, Hu J, Ma J, Yu X, Sun Y. Tumor-associated macrophages induce invasion and poor prognosis in human gastric cancer in a cyclooxygenase-2/MMP9dependent manner. American Journal of Translational Research 2019; 11(9): 6040.https://doi.org/PMID: 31632572

38. Zhang Q, Li H, Mao Y, Wang X, Zhang X, Yu X, Tian J, Lei Z, Li C, Han Q. Apoptotic SKOV3 cells stimulate M0 macrophages to differentiate into M2 macrophages and promote the proliferation and migration of ovarian cancer cells by activating the ERK signaling pathway. International journal of molecular medicine 2020; 45(1): 10-22.https://doi.org/10.3892/ijmm.2019.4408

39. Tian Y, Guo Y, Zhu P, Zhang D, Liu S, Tang M, Wang Y, Jin Z, Li D, Yan D. TRIM59 loss in M2 macrophages promotes melanoma migration and invasion by upregulating MMP-9 and Madcam1. Aging (Albany NY) 2019; 11(19): 8623.https://doi.org/10.18632/aging.102351

40. Chow A, Zhou W, Liu L, Fong MY, Champer J, Van Haute D, Chin AR, Ren X, Gugiu BG, Meng Z. Macrophage immunomodulation by breast cancer-derived exosomes requires Toll-like receptor 2mediated activation of NF-KB. Scientific reports 2014; 4(1): 1-11.https://doi.org/10.1038/srep05750

41. Liang Z-x, Liu H-s, Wang F-w, Xiong L, Zhou C, Hu T, He X-w, Wu X-j, Xie D, Wu X-r. LncRNA RPPH1 promotes colorectal cancer metastasis by interacting with TUBB3 and by promoting exosomesmediated macrophage M2 polarization. Cell death \& disease 2019; 10(11): 117.https://doi.org/10.1038/s41419-019-2077-0

\section{Figures}




\section{Figure 1 not provided with this version.}

Figure 1

MG63-Exo promoted the M2 phenotype differentiation of induced-macrophages. A: the morphology of hFOB-Exo and MG63-Exo as observed under TEM; B: the size and density distribution of hFOB-Exo and MG63-Exo particles were detected using NanoSight size analyzer; $C$ : the expression of exosomes specific marker such as CD9, CD81, and CD63 was detected by Western blot; D: immunofluorescence showed that PKH67-labeled exosomes were phagocytized by macrophages; $\mathrm{E}$ : the morphology alteration of macrophages after THP-1 induced by PMA; F: the expression of macrophage marker CD68 as detected by qRT-PCR; G: the expression of M1 and M2 macrophage markers as detected by qRT-PCR following treatment with PBS, hFOB-Exo or MG63-Exo; $\mathrm{H}$ : the effect of macrophages on the expression of M2 type cytokines TGF- $\beta$, IL-10, and VEGF as detected by qRT-PCR following treatment with PBS, hFOB-Exo or MG63-Exo; ${ }^{\mathrm{P}}<0.05$, ** $\mathrm{P}<0.01$.

\section{Figure 2 not provided with this version.}

Figure 2

MG63-Exo promoted the migration, invasion, and EMT of osteosarcoma cells through induction of M2 phenotype differentiation of macrophages. A: A schematic diagram of co-culture system in vitro; $B$ : the effects of macrophages induced by MG63-Exo on the migration and invasion of MG63 osteosarcoma cells as detected by Transwell co-culture system in vitro; C: the effect of macrophages induced by MG63Exo on the EMT of osteosarcoma cells MG63 as detected by Western blot assay. ${ }^{*} \mathrm{P}<0.05$.

\section{Figure 3 not provided with this version.}

Figure 3 
Overexpression of Tim-3 protein in MG63-Exo could be transferred to macrophages by exosomes. A: the expression of Tim-3 protein in osteosarcoma and para-carcinoma tissues as detected by Western blot assay $(n=6)$; B: the expression analysis of Tim-3 in 18 pairs of osteosarcoma and para-carcinoma tissues; $\mathrm{C}$ : the expression of Tim-3 mRNA in MG63 and hFOB as detected by qRT-PCR; D: the expression of Tim-3 protein in MG63 and hFOB as detected by Western blot assay; E: the expression of Tim-3 protein in hFOB-Exo or MG63-Exo was detected by Western blot assay; $F$ : the expression of Tim-3 protein in macrophages following treatment with PBS, hFOB-Exo or MG63-Exo as detected by Western blot; G: RTPCR was used to detect the expression of Tim-3 mRNA following the Tim-3 gene is knocked out in MG63 cells; $\mathrm{H}$ : Western blot assay was used to detect the expression of Tim-3 protein after knocking out the Tim-3 gene in MG63 cells; l: the size and density distribution of Tim-3 KO-Exo particles as detected by NanoSight size analyzer; $\mathrm{J}$ : the expression of exosome marker including, CD9, CD81, and CD63 in Tim-3 KO-Exo as detected by Western blot assay; K: the expression of Tim-3 protein in NC-Exo, MG63-Exo, and Tim-3 KO-Exo as detected by Western blot; $\mathrm{L}$ : the expression of Tim-3 protein in macrophages treated with NC-Exo, MG63-Exo and Tim-3 KO-Exo as detected by Western blot assay. ${ }^{*} \mathrm{P}<0.05,{ }^{* *} \mathrm{P}<0.01$.

\section{Figure 4 not provided with this version.}

Figure 4

Tim-3 derived from MG63-Exo promoted the migration, invasion, and EMT of osteosarcoma cells by promoting $\mathrm{M} 2$ macrophage polarization. A: the expression of $\mathrm{M} 1, \mathrm{M} 2$ macrophage marker as detected by qRT-PCR following treatment with NC-Exo, MG63-Exo or Tim-3 KO-Exo; B: the expression of M2 type cytokines TGF- $\beta$, IL-10, and VEGF in macrophage as detected by qRT-PCR following treatment with NCExo, MG63-Exo or Tim-3 KO-Exo; C: the effects of macrophages induced by NC-Exo, MG63-Exo or Tim-3 KO-Exo on the migration and invasion ability of osteosarcoma cells MG63 as determined by Transwell assay; D: the effect of macrophages induced by NC-Exo, MG63-Exo or Tim-3 KO-Exo on EMT of osteosarcoma cells MG63 as detected by Western blot. * $P<0.05$.

\section{Figure 5 not provided with this version.}

Figure 5 
Tim-3 derived from MG63-Exo promoted lung metastasis of osteosarcoma cells in nude mice by promoting M2 macrophage polarization. * $\mathrm{P} \llbracket 0.05$. 